\title{
Robust Collaboration of a Haptically-Enabled Double-Slave Teleoperation System under Random Communication Delays
}

\begin{abstract}
Addressing stability and performance issues due to communication delays in multilateral teleoperation systems, this paper proposes a robust control strategy for synchronisation of such systems. Multilateral teleoperation configurations usually include more than two communication channels, and therefore, face sever latencies and uncertainties in data transmission routes. This study develops a sliding surface based on the synchronization errors characterized between each ends of the considered multilateral teleoperation system. Here, two slave robots receive commands from the master system to cooperatively execute the desired teleoperation task in the remote, shared workspace. Lyapunov stability analysis approach guarantees the performance of the proposed controller. Moreover, the effectiveness of the controller is experimentally evaluated through a real-world Internet-based double-slave teleoperation system.

Index Terms-Sliding mode, robust control, multi-robot, teleoperation, time-varying delay.
\end{abstract}

\section{INTRODUCTION}

Multilateral teleoperation systems have critical applications, from medical surgery [1]-[4] to space explorations [5]-[8]. In all of those applications, dealing with uncertainties and disturbances in communication between the two main parts of the teleoperation has been always challenging, as it threatens the stability and performance of the teleoperation task in the remote workspace [9]. Researchers have been investigating several approaches to tackle this problem, specifically for multilateral teleoperation [10], [11], such as predictive simulator [12] Petri-net [13], central control [14], adaptive nonlinear methods [15]-[18], and learning techniques [19]-[22], to name a few. However, some multi-robot-specific objectives, like collision avoidance and kinematic synchronizations between the cooperative robots, yet need further investigations.

On the other hand, these challenges will get much problematic in the presence of random communication delays between the master and slave sides of a teleoperation system. Timedelays will disturb the signals being transferred between the master-slave sites. Consequently, stability and performance of the system is jeopardized, and therefore, more chance for failure, damage, and collision.

Several studies have been conducted to tackle the aforementioned issues. Synchronizing the slave robots based on their common object tool motion is one of the famous approaches but with a limited applicability. The problem with this approach is that it does not consider the internal forces between the robots' end-effectors. Leader-follower strategy is another approach that takes one of the robots as the leader subsystem and the other ones follow the commands distributed by the leader. The leader is in communication with the master side and receives the teleoperation task commands from the system. This approach is appropriate for internal force considerations, however, synchronizing the slave systems locally is still challenging.

Some other research have proposed control solutions for multilateral teleoperation systems, however, no uncertainty is taken into account such as [23]. Developed a control methodology for a multi-slave teleoperation, [24] did not deal with network uncertainties neither kinematic constraints between the slave robots. Collision between any of slave systems, tools, and objects in the remote environment is the main kinematic constrain that should be critically considered in the control design for such systems. [25]-[27] have considered with this constraint in their studies, however, communication delays and internal forces were not dealt with. Internal forces are dynamic constraints between the slave robots that should be also taken care of. These constraints do not interfere with the motions in the remote workspace. [28]-[30] have developed control strategies for this constraint, however, they some needed a human operator to monitor the performance, or very accurate information of the system which is not practically feasible.

These limitations are the main motivation that drives this research. Hence, this article investigates and proposes a novel robust solution. The main contributions of the paper are listed as follows.

- a single-master dual-slave experimental teleoperation setup is established over the real-world Internet-based communication network. Considering the time-varying delayed control command and haptic signals, dynamics of the multilateral teleoperation system is fully described in mathematical terms.

- Defining a sliding surface for synchronization errors, this study proposes a robust control algorithm for the dualslave teleoperation system. The proposed control strategy guarantees the stable performance of the collaborative teleoperation process while tackling random delays and disturbances through the communication network.

- Moreover, the proposed framework provides the human operator with a haptic signal that indicates the balanced/unbalanced positioning of the object being handled by the slave robots in the remote workspace.

Additionally, effectiveness of the proposed solution is evalu- 
ated on the real-world Internet-based multilateral teleoperation system considered in this study.

The paper is organized as follows; after describing the dynamical equations of motion of the system in Section II, Section III proposes the robust synchronization algorithm and explains its derivation. Section IV demonstrates the experimental simulation results, followed by conclusion remarks and future directions discussed in Section V.

\section{Multilateral Teleoperation Systems DESCRIPTION}

This section presents the mathematical model of dynamics of the multilateral teleoperation system illustrated in Figure 1. Here, the considered multilateral teleoperation system consists of one master device being controlled by the human operator, an Internet-based communication network, and two slave subsystems operating in the remote workspace (Figure 1a). The slave manipulators are supposed to shift the heavy circular shape object shown in Figure 1 from the back end of the work table to the front end. This process is further explained in Section IV. The slave systems receive control commands from the master device. The human operator should be feeling the attachment and weight of the object while the slave manipulators handling it. Figure 1b shows the master console that the human operator commands the desired task through the haptic device providing the force feedback.

\section{A. Master Haptic System}

The haptic device considered as the master system has 6 degrees of freedom in its Cartesian workspace $\vec{x}_{m}=$ $\left[x_{m}, y_{m}, z_{m}, \alpha_{m}, \beta_{m}, \gamma_{m}\right]^{T} \in \mathbb{R}^{6 \times 1}$. However, in it has only three actuated joints $\left[q_{m 1}, q_{m 2}, q_{m 3}\right]^{T} \in \mathbb{R}^{3 \times 1}$ and three passive gimbals joints $\left[q_{m 4}, q_{m 5}, q_{m 6}\right]^{T} \in \mathbb{R}^{3 \times 1}$, and in total $q_{m}=\left[q_{m 1}, q_{m 2}, q_{m 3}, q_{m 4}, q_{m 5}, q_{m 6}\right]^{T} \in \mathbb{R}^{6 \times 1}$. Equations of motion of the master haptic device are:

$$
M_{m} \ddot{x}_{m}+C_{m}\left(x_{m}, \dot{x}_{m}\right) \dot{x}_{m}+g_{m}\left(x_{m}\right)=\tau_{m}-\left[\begin{array}{llll}
f_{h}^{T} & 0 & 0 & 0
\end{array}\right]^{T}
$$

where, $M_{m} \in \mathbb{R}^{6 \times 6}$ is the inertia matrix, $C_{m}\left(x_{m}, \dot{x}_{m}\right) \in$ $\mathbb{R}^{6 \times 6}$ is the Centripetal and Coriolis matrix, and $g_{m}\left(x_{m}\right) \in$ $\mathbb{R}^{6 \times 1}$ is the potential vector of the master device. $\tau_{m} \in \mathbb{R}^{6 \times 1}$ is the control signals commanded on the master device's actuators. Notably, the first three elements of $\tau_{m}$ are applied by device's actuators to provide the three dimensional Cartesian force (haptic) elements on the human operator's hand. These haptic terms are in interaction with $f_{h} \in \mathbb{R}^{3 \times 1}$. While, the last three elements of $\tau_{m}$ are imposed only by the human operator.

\section{B. Dual-slave Subsystem}

Every slave's dynamics is similarly modelled as:

$$
\left\{\begin{array}{l}
\mathrm{M}_{s 1} \ddot{x}_{s 1}+\mathrm{C}_{s 1} \dot{x}_{s 1}+\mathrm{g}_{s 1}=\tau_{s 1}-f_{e 1} \\
\mathrm{M}_{s 2} \ddot{x}_{s 2}+\mathrm{C}_{s 2} \dot{x}_{s 2}+\mathrm{g}_{s 2}=\tau_{s 2}-f_{e 2}
\end{array}\right.
$$

where, $f_{e 1,2} \in \mathbb{R}^{3 \times 1}$ are the force vectors from each slave robot interacting within the remote environment. It should be noted that the matrix/vector coefficients $\mathrm{M}_{s 1,2} \in \mathbb{R}^{3 \times 3}$, $\mathrm{C}_{s 1,2} \in \mathbb{R}^{3 \times 3}$, and $\mathrm{g}_{s 1,2} \in \mathbb{R}^{3 \times 1}$ are all derived in the

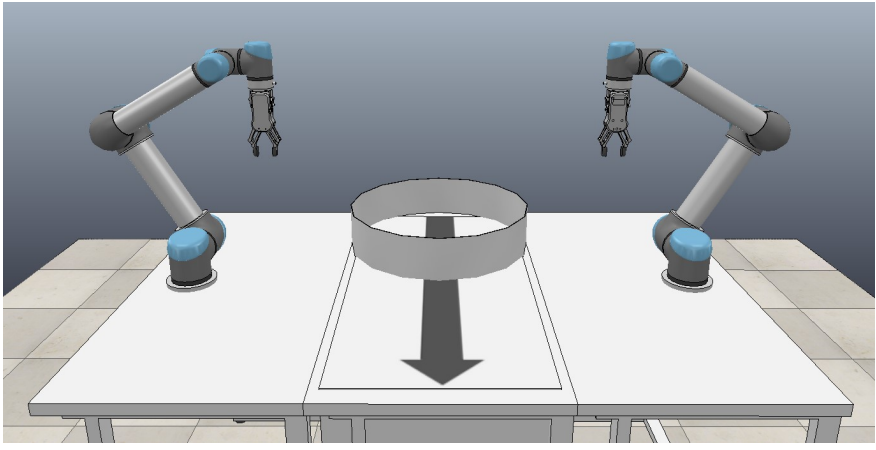

(a) The slave robots in V-REP simulation [31].

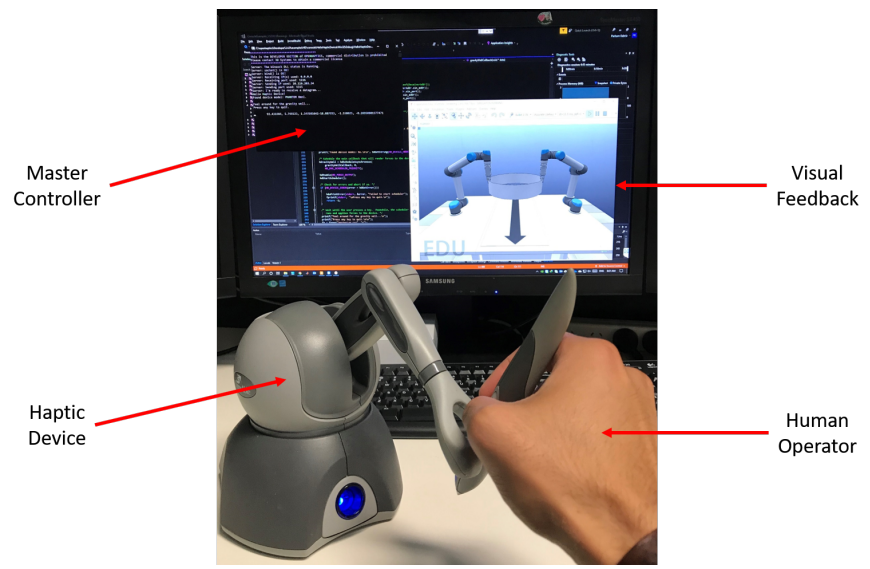

(b) The master console.

Fig. 1. (a) Slave workspace of the considered dual-slave multilateral teleoperation system. Two UR5 manipulators are considered as slave subsystems that are desired to stably grasp and carry an object in a cooperative regime. (b) Master console of the considered multilateral teleoperation system, including a haptic device, master controller and visualization (a desktop computer).

Cartesian workspace since $\ddot{x}_{s 1,2} \in \mathbb{R}^{3 \times 1}, \dot{x}_{s 1,2} \in \mathbb{R}^{3 \times 1}$, and $x_{s 1,2} \in \mathbb{R}^{3 \times 1}$, are acceleration, velocity and position vectors of the slave robots, respectively.

\section{Communication Network}

Communication protocol plays an important role in teleoperation systems; Command signals transmitted from master to slave, and sensory information, in particular haptic signals, fed back to the master side. However, the transmitted reference are asymmetrically interrupted by forward and backward delays:

$$
\begin{aligned}
& x_{s}^{*}(t)=x_{m}\left(t-d_{f}(t)\right) \\
& f_{h}^{*}(t)=f_{e}\left(t-d_{b}(t)\right)
\end{aligned}
$$

Latencies and uncertainties through the communication medium have negative impacts on stability, quality, and performance of the teleoperation process. Considering these factors in our controller design, we make two assumptions on the delay functions as below:

1) boundedness of the delay: $\lim _{t \rightarrow+\infty}\left(t-d_{f, b}(t)\right)=+\infty$

2) continuously differentiable with $\left|\dot{d}_{f, b}(t)\right|<1, \forall t>0$

These assumptions are not far from real-world occasions, as most of the researchers have previously verified their validity 
in the literature [9]. Moreover, we have also experienced and presented the Internet-based communication quality in the next sections that validates these assumptions in practice.

\section{Teleoperation Workspace}

Taking into account a dynamic behaviours of the remote environment that the slave robots working in, we consider a non-homogeneous mass-spring-damper model of an object that the slave robots collaboratively interacting with. This model is expressed as:

$$
M_{e} \ddot{x}_{e}+D_{e} \dot{x}_{e}+S_{e} x_{e}=f_{e}+f_{E}
$$

with $M_{e} \in \mathbb{R}^{3 \times 3}, D_{e} \in \mathbb{R}^{3 \times 3}$, and $S_{e} \in \mathbb{R}^{3 \times 3}$ as the mass, damping, and spring coefficients, respectively. $\ddot{x}_{e}, \dot{x}_{e}$, and $x_{e}$ are the acceleration, velocity, and position of the object in the Cartesian workspace, respectively. $f_{e}$ is the non-homogeneous term, while $f_{E}$ is the external force imposed by the slave manipulators on the object. IT should be noted that the object's position $x_{e}$ is calculated on its centre of mass and is considered as the remote centre of motion (RCM). To successful execution of the teleoperation task, Cartesian position of the slave robots' end-effector should always maintain the desired position and velocity that satisfy the Jacobian relation:

$$
\dot{x}_{e}=J_{e 1} \dot{x}_{s 1}=J_{e 2} \dot{x}_{s 2}
$$

where, $J_{e 1,2}$ are the Jacobian matrices translating Cartesian velocity of each slave end-effector to RCM. This relation is to maintain the relative positions and velocities between the slaves' end-effectors and RCM. To achieve this goal, a set of kinematic constraints should be considered and met during the task execution:

$$
\left\{\begin{array}{l}
\phi_{s 1}^{T} x_{s 1}+\phi_{s 2}^{T} x_{s 2}+\phi_{e}^{T} x_{e}=0 \\
\psi_{s 1}^{T} \dot{x}_{s 1}+\psi_{s 2}^{T} \dot{x}_{s 2}+\psi_{e}^{T} \dot{x}_{e}=0 \\
\chi_{s 1}^{T} \ddot{x}_{s 1}+\chi_{s 2}^{T} \ddot{x}_{s 2}+\chi_{e}^{T} \ddot{x}_{e}=0
\end{array}\right.
$$

in which, the coefficient vectors $\phi_{\{s 1, s 2, e\}}, \psi_{\{s 1, s 2, e\}}$, and $\chi_{\{s 1, s 2, e\}}$ are all non-zero. This means that Cartesian position, velocity, and acceleration of the slaves' end-effector and RCM should have no null-space and cannot be singular with respect to each other. Moreover, equation 6 indicates a linear relationship between the corresponding Cartesian variables.

Additionally, This kinematic constraints introduce a redundant relationship between the forces being applied by the slave end-effectors. This redundancy is a very useful, yet challenging feature in cooperative tasks, such as tension and torsion between the robots and objects in the remote workspace. To cope and utilize this feature the force imposed on the every slave robot is considered as:

$$
f_{E}=J_{e i}^{\dagger} f_{e}+\left(I-J_{e i}^{\dagger} J_{e i}\right) \zeta \quad, \quad i=1,2
$$

where $\zeta$ presents the null-space that additional cooperative goals could be achieved in. To comply with (7) and being able to accomplish some secondary tasks in $\zeta$, the leader follower strategy is suggested [32]. In other words,

$$
\zeta=\left[-\frac{1}{S-1} I_{s 1}, \ldots,-\frac{1}{S-1} I_{s S}\right]^{T} f_{l e a d}
$$

$I$ is the identity matrix and $S$ is the total number of slave robots, which, in our case is 2 , and $f_{\text {lead }}$ is the force of the selected leader robot. Therefore,

$$
\zeta=\left[-I_{s 1},-I_{s 2}\right]^{T} f_{\text {lead }}
$$

We use this methodology to develop our controller and synchronisation mechanism in the following section.

\section{Robust Collaboration of Single-master DUAL-SLAVE TELEOPERATION SYSTEMS}

This section derives a robust control algorithm for the considered single-master dual-slave teleoperation configuration. The control algorithm is according to the models and assumptions made in the previous section. First of all, we employ the filtering approach introduced in [16] to generate smooth estimations of the perturbed reference signals in (3). Therefore, the controllers at each side of the teleoperation system will have a smoothed reference command signal. In more details, the desired position of the slave robots which are commanded to handle an object in the remote environment, for example, will be smoothly approximated by:

$$
\begin{aligned}
& \text { slave\#1: }\left\{\begin{array}{l}
\dot{\hat{x}}_{s 1}=\xi_{s 1}+c_{s 11} c_{s 12}\left(x_{s 1}^{*}-\hat{x}_{s 1}\right) \\
\dot{\xi}_{s 1}=c_{s 11}^{2} c_{s 13}\left(x_{s 1}^{*}-\hat{x}_{s 1}\right)
\end{array}\right. \\
& \text { slave\#2: }\left\{\begin{array}{l}
\dot{\hat{x}}_{s 2}=\xi_{s 2}+c_{s 21} c_{s 22}\left(x_{s 2}^{*}-\hat{x}_{s 2}\right) \\
\dot{\xi}_{s 2}=c_{s 21}^{2} c_{s 23}\left(x_{s 2}^{*}-\hat{x}_{s 2}\right)
\end{array}\right.
\end{aligned}
$$

for the slave robots, where, $\hat{x}_{s 1}$ and $\hat{x}_{s 2}$ are the smoothly estimated positions for the first and second slave manipulators, respectively. It should be noted that the reference desired positions $x_{s 1}^{*}$ and $x_{s 2}^{*}$ are deterministically derived via some mathematical manipulations according to the discussions on RCM (5)-(6) in the previous section. Notably, that derivation is not an objective in this study, and moreover, it has been well-addressed in the literature (for instance, see [24], [25]).

On the other side, reference signals, mainly haptic $\left(f_{h}^{*}\right)$, for the master device is also estimated by $\hat{f}_{h}$ :

$$
\left\{\begin{array}{l}
\dot{\hat{f}}_{h}=\xi_{m}+c_{m 1} c_{m 2}\left(f_{h}^{*}-\hat{f}_{h}\right) \\
\dot{\xi}_{m}=c_{m 1}^{2} c_{m 3}\left(f_{h}^{*}-\hat{f}_{h}\right)
\end{array}\right.
$$

in either (9) or (10), $c_{(.)} \mathrm{s}$ are constant parameters to be designed in later sections. These constants determine the smoothness and effort of the estimating filters. Therefore, they can be obtained by either trial and error or numerical simulations. Although they might have effects on the stability of the overall system, this matter could be simply addressed by a proper selection of these parameters and it has been extensively discussed in [16] and references therein. The main consideration regarding these parameters is that they should be chosen in a way to make the polynomial $\eta^{2}+c_{(.) 1} \eta+c_{(.) 2}$ Hurwitz with respect to $\eta$. The third one, $c_{(.) 3}$, only needs to be positive.

Practical and precise model of a system under consideration is a necessity to design an effective controller for the system. However, it is not usually easy to derive such a model due 
to non-linearities and complexities often exist in physical systems. To overcome this difficulty we need to estimate systems' behaviours. Dynamics of the robotic systems have a property called linear-in-parameters [24], which is generally expressed as:

$$
M \ddot{x}+C(x, \dot{x}) \dot{x}+g(x)=\Psi(x, \dot{x}, \ddot{x}) \theta
$$

in which, $\theta$ is the parameters vector of the system, which introduces major uncertainties to the system. Hence, developing a parametrised structure $\Psi(.) \hat{\theta}$ similar to right hand side of (11) in the controller can help in tackling parameter uncertainties of the system and improving the stability and performance of the desired task. This property of robotic systems is actually a great tool in development of adaptive control algorithms for such systems, especially teleoperation applications [9]. To achieve this goal, the following theorem proposes a robust synchronisation mechanism for the considered type of teleoperation systems.

a) Theorem: Having smooth reference signals $\hat{x}_{s i}$ and $\hat{f}_{h}$ ( $i=1,2$ for the two slaves), provided by (9) and (10), the controllers:

$$
\begin{aligned}
\tau_{s i} & =M_{s i}\left(c_{s i 1}^{2} c_{s i 3}\left(x_{s i}^{*}-\hat{x}_{s i}\right)+Q_{s i}\left(\dot{\hat{x}}_{s i}-\dot{x}_{s i}\right)\right) \\
& +C_{s i}\left(\xi_{s i}+Q_{s i}\left(\hat{x}_{s i}-x_{s i}\right)\right)+g_{s i} \\
& -P_{s i}\left(\dot{x}_{s i}-\xi_{s i}+Q_{s i}\left(\hat{x}_{s i}-x_{s i}\right)\right) \\
& -R_{s i} S_{s i}-K_{s i} \operatorname{sat}\left(S_{s i}\right)+\Psi_{s i} \hat{\theta}_{s i}+\hat{f}_{E}
\end{aligned}
$$

with the sliding surface:

$$
\text { slaves: }\left\{\begin{array}{l}
\tilde{x}_{s i}=x_{s i}-\hat{x}_{s i} \\
\dot{\tilde{x}}_{s i}=\dot{x}_{s i}-\zeta_{s i} \\
S_{s i}=\dot{\tilde{x}}_{s i}+P_{s i} \tilde{x}_{s i}
\end{array}\right.
$$

for the slave systems, and

$$
\tau_{m}=\hat{f}_{h}+g_{m}-x_{m}-P_{m}\left(\dot{x}_{m}+x_{m}\right)
$$

and

$$
S_{m}=\dot{x}_{m}+P_{m} x_{m}
$$

for the master system, where $P_{(.)}, Q_{(.)}, R_{(.)}$, and $K_{(.)}$are symmetric positive definite design matrices, and sat(.) is the saturation function, guarantee the uniform stability of the systems (1) and (2) with the smoothing filters (9)-(10), and environmental dynamics (4).

b) Proof: First, we focus on the slave systems. Considering (11) and (13), substituting the $\tau_{s i}$ in (2) by (12), it results in

$$
\begin{array}{r}
M_{s i} \dot{S}_{s i}+\left(C_{s i}+Q_{s i}+R_{s i}\right) S_{s i}+K_{s i} \operatorname{sat}\left(S_{s i}\right)= \\
\Psi_{s i}\left(\theta_{s i}-\hat{\theta}_{s i}\right)-\left(f_{E}-\hat{f}_{E}\right)
\end{array}
$$

in which, the estimation errors will be rewritten as $\tilde{f}_{E}=$ $f_{E}-\hat{f}_{E}$ and $\tilde{\theta}_{s i}=\theta_{s i}-\hat{\theta}_{s i}$. The next step is to proposing a Lyapunov candidate function:

$$
V_{s i}=\frac{1}{2}\left(S_{s i}^{T} M_{s i} S_{s i}+\tilde{\theta}_{s i}^{T} \Gamma_{s i} \tilde{\theta}_{s i}+\tilde{f}_{E}^{T} \Lambda_{s i} \tilde{f}_{E}\right)
$$

where $\nu^{T} \Gamma_{s i} \nu>0$ and $\nu^{T} \Lambda_{s i} \nu>0, \forall \nu \in \mathbb{R}^{n \times 1}$. The next step is to differentiate the Lyapunov function alongside the systems trajectories and verify its negativeness:

$$
\begin{aligned}
\dot{V}_{s i}= & -S_{s i}^{T}\left(Q_{s i}+R_{s i}\right) S_{s i}-S_{s i}^{T} K_{s i} \operatorname{sat}\left(S_{s i}\right) \\
& +\left(S_{s i}^{T} \Psi_{s i}+\dot{\tilde{\theta}}_{s i}^{T} \Gamma_{s i}\right) \tilde{\theta}_{s i}-\left(S_{s i}^{T}-\dot{\tilde{f}}_{E}^{T} \Lambda_{s i}\right) \tilde{f}_{E}
\end{aligned}
$$

It should be noted that $S_{s i}^{T}\left(\dot{M}_{s i}-2 C_{s i}\right) S_{s i}=0$ due to the skew-symmetry property of (11) [14] $\dot{\tilde{\theta}}_{s i}$ and $\dot{\hat{f}}_{E}$ are the estimation mechanisms that should be designed in a way to make $\dot{V}_{s i}$ negative, which are proposed as:

$$
\dot{\tilde{\theta}}_{s i}=-\Gamma_{s i}^{-1} \Psi_{s i} S_{s i} \quad, \quad \dot{\tilde{f}}_{E}=\Lambda_{s i}^{-1} S_{s i}
$$

that will result in

$$
\dot{V}_{s i}=-S_{s i}^{T}\left(Q_{s i}+R_{s i}\right) S_{s i}-S_{s i}^{T} K_{s i} \operatorname{sat}\left(S_{s i}\right)
$$

which is negative by a proper selection of the function sat(.). Knowing that $V_{s i}$ is lower-bounded and $\dot{V}_{s i}$ is uniformly continuous, it means that [14]:

$$
\lim _{t \rightarrow+\infty} \dot{V}_{s i}=0
$$

which also indicates the asymptotic stability of the slave systems.

On the other hand, considering the following Lyapunov candidate function for the master system:

$$
V_{m}=\frac{1}{2}\left(S_{m}^{T} M_{m} S_{m}+x_{m}^{T} x_{m}+\tilde{f}_{h}^{T} \tilde{f}_{h}\right)
$$

then, differentiation along time:

$$
\dot{V}_{m}=\frac{1}{2} S_{m}^{T} \dot{M}_{m} S_{m}+S_{m}^{T} M_{m} \dot{S}_{m}+x_{m}^{T} \dot{x}_{m}+\tilde{f}_{h}^{T} \dot{\tilde{f}}_{h}
$$

which, by considering (1) and (15), the skew-symmetry property, and then, substituting (14) results in

$$
\dot{V}_{m}=-x_{m}^{T} P_{m} x_{m}+\tilde{f}_{h}^{T}\left(\dot{\tilde{f}}_{h}-S_{m}\right)
$$

which by proposing the adaptive law:

$$
\dot{\tilde{f}}_{h}=S_{m}=\dot{x}_{m}+P_{m} x_{m}
$$

the remaining $-x_{m}^{T} P_{m} x_{m}$ is negative and implies the uniform stability of the master system, and this completes the proof.

c) Remark: It should be noted that the master device is being controlled by the human operator and once it is settled in a position commanded by the operator, $x_{m}=x_{\text {human }}$, the system is asymptotically stable $\dot{x}_{m}=0$. Moreover, The adaptation law (24) also complies with the sliding surface (15). In other words, the force tracking is simultaneously achieved while the system is stably following the human operator's commands. Robustness and effectiveness of the proposed synchronization algorithm is being demonstrated in the next section. 

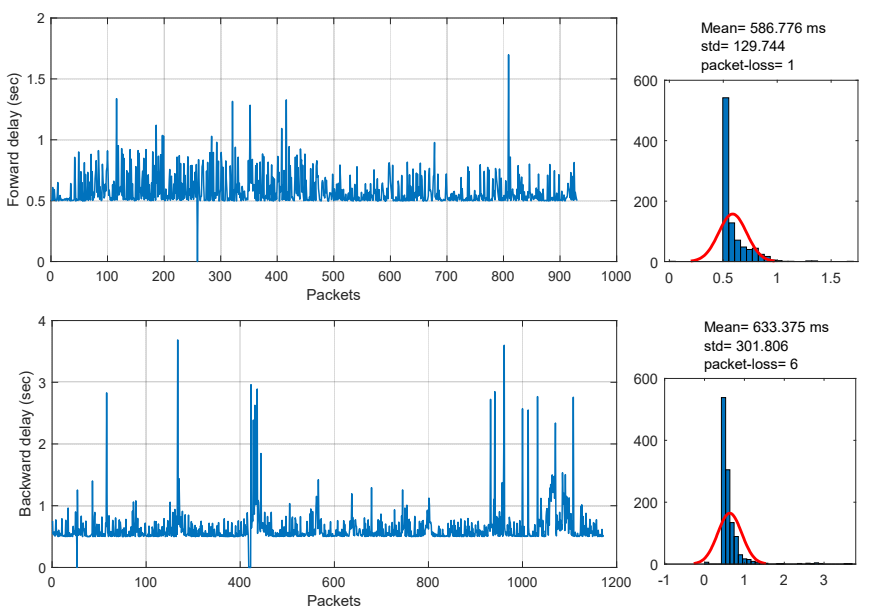

Fig. 2. Forward and backward delays through the wireless network between the master and slave systems.
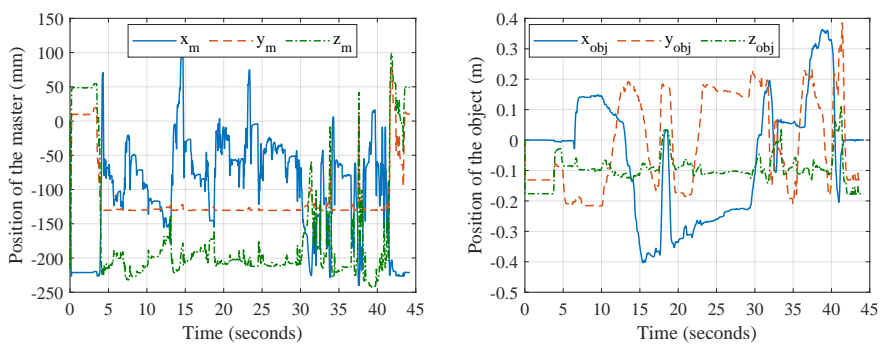

Fig. 3. Positions of the master device and the object in the remote workspace.

\section{EXPERIMENTAL RESULTS AND DISCUSSIONS}

In this section, we evaluate and demonstrate the performance of the proposed control methodology. The teleoperation setup shown in Figure 1 is considered for the evaluation purposes. The communication between the master and slave sides are measured and reported in Figure 2. It should be mentioned that the considered object weighs $5 \mathrm{~kg}$ which is relatively heavy considering the maximum capacity of UR5 manipulators. On the other side, shape and size of the object is quite large and difficult to balance. This is to purposefully impose unbalanced loads on each slaves. As a result, asymmetrical forces are observed in force sensors, and consequently, haptic signals.

Figure 3 shows the Cartesian position of the master device handled by the human operator and the object in the remote workspace. Orientations of the master and object are also shown in Figure 4. Noticeably, these signals are not quite similar as there is a scaling and transformation between the systems due to differences in the corresponding workspaces. However, these figures illustrating the robust and stable position tracking of the slave systems. Moreover, Figure 5 depicts the effective force reflection of the proposed control algorithm that also provides the synchronisation of the two slave robots while commanded from the master side for grasping, lifting, moving, and placing the object in the remote environment.
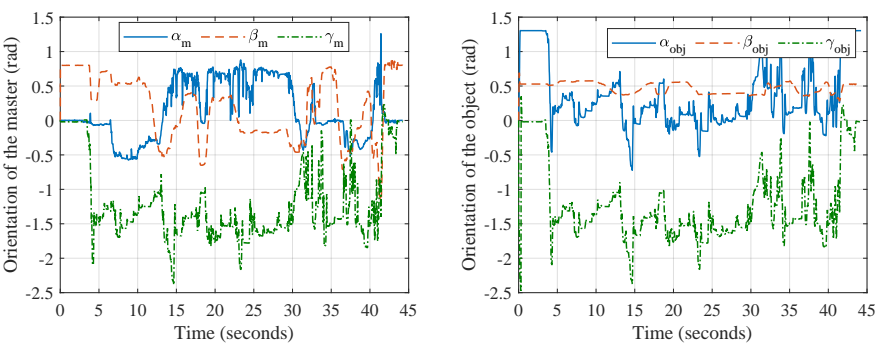

Fig. 4. Orientations of the master device and the object in the remote workspace.
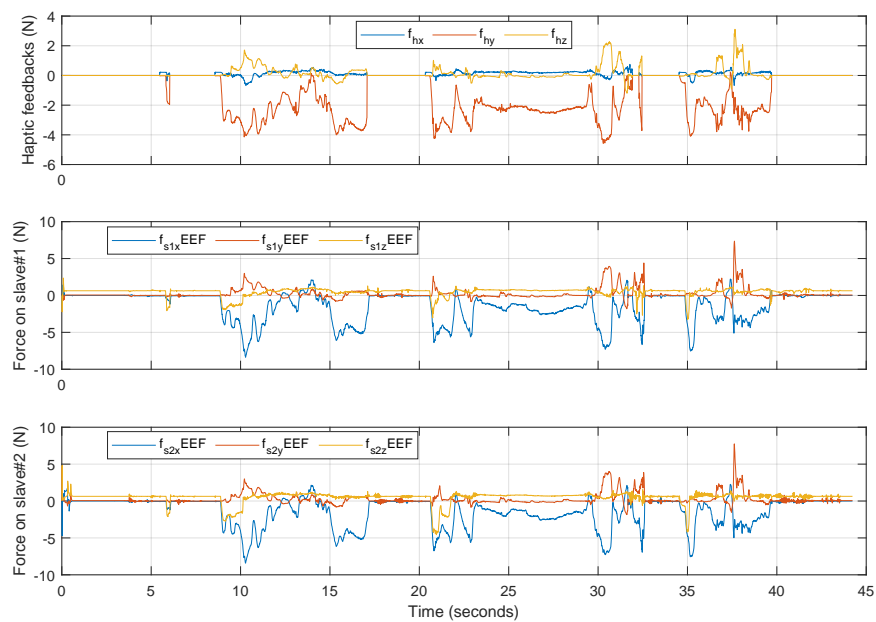

Fig. 5. Haptic signals on the master device and force measurements of the slave robots in the remote workspace.
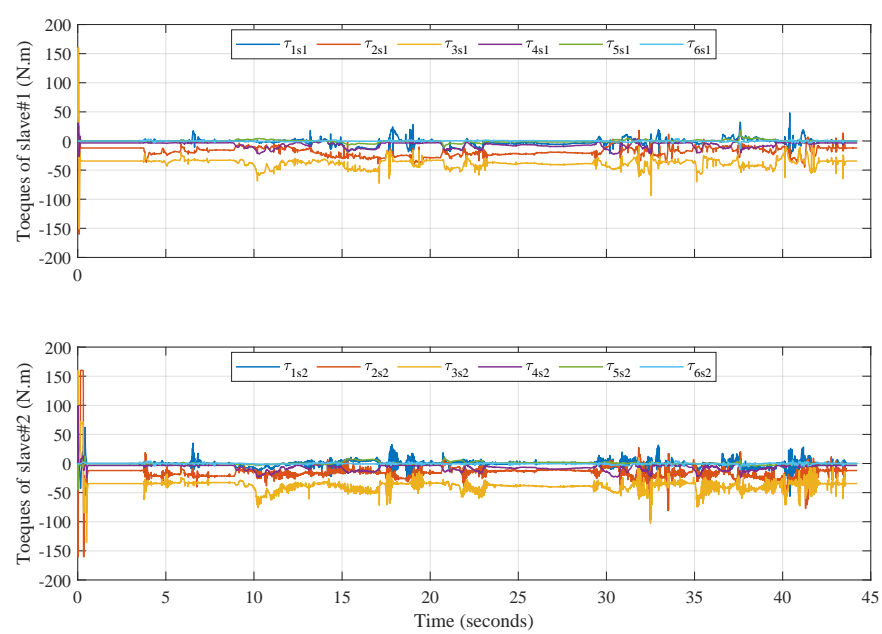

Fig. 6. Control torques of the slave robots.

The main element in force reflection is along the vertical $(z)$ axis, which is against the gravity and indicates the weight of the object to be carried by the slave robots. Comparison between the three figures in Figure 5 implies the main advantage of the proposed synchronisation methodology that is providing the human operator with the sense of the unbalanced load in the remote workspace while controlling multiple slave 
robots with only one haptic device. Figure 6 also shows the control torques generated by the proposed approaches (12). Insignificant difference between the two groups of signals $\tau_{s 1}$ and $\tau_{s 2}$ indicates the effort of each controller to compensate for asynchronous motions and loads.

\section{CONCLUSIONS AND Future Work}

Single-master multiple-slave teleoperation systems are employed for more difficult tasks to be cooperatively executed in a remote workspace. In addition to communication uncertainties and latencies induced by the network, stable synchronization of multiple slave systems is a challenge that researchers have been working on for years. Utilizing smooth filtering and sliding mode techniques, this paper proposed a robust synchronization strategy for a single-master dual-slave teleoperation system. The proposed approach has been theoretically analysed and experimentally verified. Furthermore, it has also outperformed two highly cited previous solutions in a comparative examination.

Future perspectives of this work can lead to a semiautonomous multilateral teleoperation configurations that can accomplish more complicated tasks.

\section{REFERENCES}

[1] G. T. Sung and I. S. Gill, "Robotic laparoscopic surgery: a comparison of the da vinci and zeus systems," Urology, vol. 58, no. 6, pp. 893-898, 2001.

[2] Q. Miao, A. McDaid, M. Zhang, P. Kebria, and H. Li, "A threestage trajectory generation method for robot-assisted bilateral upper limb training with subject-specific adaptation," Robotics and Autonomous Systems, vol. 105, pp. 38-46, 2018.

[3] M. M. Dalvand, S. Nahavandi, M. Fielding, J. Mullins, Z. Najdovski, and R. D. Howe, "Modular instrument for a haptically-enabled robotic surgical system (herosurg)," IEEE Access, vol. 6, pp. 31974-31982, 2018.

[4] T. Haidegger, "Autonomy for surgical robots: Concepts and paradigms," IEEE Transactions on Medical Robotics and Bionics, vol. 1, no. 2, pp. 65-76, 2019.

[5] P. Putz, "Space robotics in europe: A survey," Robotics and Autonomous Systems, vol. 23, no. 1-2, pp. 3-16, 1998.

[6] L. Pedersen, D. Kortenkamp, D. Wettergreen, I. Nourbakhsh, and D. Korsmeyer, "A survey of space robotics," nasa.gov, 2003.

[7] G. A. Landis, "Robots and humans: synergy in planetary exploration," Acta astronautica, vol. 55, no. 12, pp. 985-990, 2004.

[8] T. Haidegger, J. Sándor, and Z. Benyó, "Surgery in space: the future of robotic telesurgery," Surgical endoscopy, vol. 25, no. 3, pp. 681-690, 2011.

[9] P. M. Kebria, H. Abdi, M. M. Dalvand, A. Khosravi, and S. Nahavandi, "Control methods for internet-based teleoperation systems: A review," IEEE Transactions on Human-Machine Systems, vol. 49, no. 1, pp. 32 46, 2018.

[10] T. Fong, C. Thorpe, and C. Baur, "Multi-robot remote driving with collaborative control," IEEE Transactions on Industrial Electronics, vol. 50, no. 4, pp. 699-704, 2003.

[11] M. Panzirsch, R. Balachandran, and J. Artigas, "Cartesian task allocation for cooperative, multilateral teleoperation under time delay," in 2015 IEEE International Conference on Robotics and Automation (ICRA). IEEE, 2015, pp. 312-317.

[12] N. Y. Chong, T. Kotoku, K. Ohba, K. Komoriya, K. Tanie, J. Oaki, H. Hashimoto, F. Ozaki, K. Maeda, and N. Matsuhira, "A collaborative multi-site teleoperation over an isdn," Mechatronics, vol. 13, no. 8-9, pp. 957-979, 2003.

[13] I. H. Elhajj, A. Goradia, N. Xi, C. M. Kit, Y. H. Liu, and T. Fukuda, "Design and analysis of internet-based tele-coordinated multi-robot systems," Autonomous Robots, vol. 15, no. 3, pp. 237-254, 2003.
[14] S. Sirouspour, "Modeling and control of cooperative teleoperation systems," IEEE Transactions on Robotics, vol. 21, no. 6, pp. 1220-1225, 2005.

[15] P. M. Kebria, A. Khosravi, S. Nahavandi, F. Bello, and S. Krishnan, "Robust adaptive synchronisation of a single-master multi-slave teleoperation system over delayed communication," in 2019 IEEE International Conference on Industrial Technology, 2019, pp. 193-198.

[16] P. M. Kebria, A. Khosravi, S. Nahavandi, P. Shi, and R. Alizadehsani, "Robust adaptive control scheme for teleoperation systems with delay and uncertainties," IEEE Transactions on Cybernetics, pp. 1-11, 2019.

[17] P. M. Kebria, A. Khosravi, S. Nahavandi, D. Watters, G. Guest, and P. Shi, "Robust adaptive control of internet-based bilateral teleoperation systems with time-varying delay and model uncertainties," in 2019 IEEE International Conference on Industrial Technology (ICIT), 2019, pp. 187-192.

[18] P. M. Kebria, A. Khosravi, S. Nahavandi, A. Homaifar, and M. Saif, "Experimental comparison study on joint and cartesian space control schemes for a teleoperation system under time-varying delay," in 2019 IEEE International Conference on Industrial Technology (to be published), 2019, pp. 1-6.

[19] P. M. Kebria, A. Khosravi, S. Nahavandi, D. Wu, and F. Bello, "Adaptive type-2 fuzzy neural-network control for teleoperation systems with delay and uncertainties," IEEE Transactions on Fuzzy Systems, pp. 1-12, 2019.

[20] P. M. Kebria, A. Khosravi, S. Nahavandi, Z. Najdovski, and S. J. Hilton, "Neural network adaptive control of teleoperation systems with uncertainties and time-varying delay," in 2018 IEEE 14th International Conference on Automation Science and Engineering (CASE). IEEE, 2018, pp. 252-257.

[21] P. M. Kebria, A. Khosravi, S. M. J. Jalali, and S. Nahavandi, "Type2 fuzzy neural network synchronization of teleoperation systems with delay and uncertainties," in 2019 IEEE 15th International Conference on Automation Science and Engineering (CASE), Aug 2019, pp. 16251630.

[22] — "Adaptive type-2 fuzzy control scheme for robust teleoperation under time-varying delay and uncertainties," in 2019 IEEE 15th International Conference on Automation Science and Engineering (CASE), Aug 2019, pp. 1631-1636.

[23] A. Peer, S. Hirche, C. Weber, I. Krause, M. Buss, S. Miossec, P. Evrard, O. Stasse, E. S. Neo, A. Kheddar et al., "Intercontinental multimodal tele-cooperation using a humanoid robot," in 2008 IEEE/RSJ International Conference on Intelligent Robots and Systems. IEEE, 2008, pp. 405-411.

[24] D. Lee and M. W. Spong, "Bilateral teleoperation of multiple cooperative robots over delayed communication networks: Theory," in Proceedings of the 2005 IEEE international conference on robotics and automation. IEEE, 2005, pp. 360-365.

[25] B. Yao, W. Gao, S. Chan, and M. Cheng, "Vsc coordinated control of two manipulator arms in the presence of environmental constraints," IEEE Transactions on Automatic Control, vol. 37, no. 11, pp. 18061812, 1992.

[26] S. Schneider and R. H. Cannon, "Object impedance control for cooperative manipulation: Theory and experimental results," in Proceedings, 1989 International Conference on Robotics and Automation. IEEE, 1989, pp. 1076-1083.

[27] S. Nahavandi, Z. Najdovski, B. Horan, and A. Bhatti, "Method and apparatus for haptic control," Nov. 3 2015, uS Patent 9,174,344.

[28] P. Prokopiou, W. Harwin, and S. Tzafestas, "Fast, intuitive and timedelays-robust telemanipulator designs using a human arm model," in Proceedings 6th Symposium on Intelligent Robotic Systems. Edinburgh, UK, 1998, pp. 7-16.

[29] A. Bemporad, "Predictive control of teleoperated constrained systems with unbounded communication delays," in Proceedings of the 37th IEEE Conference on Decision and Control. IEEE, 1998, pp. 21332138.

[30] S. Katsura, T. Suzuyama, and K. Ohishi, "A realization of multilateral force feedback control for cooperative motion," IEEE Transactions on Industrial Electronics, vol. 54, no. 6, pp. 3298-3306, 2007.

[31] [Online]. Available: https://www.coppeliarobotics.com/

[32] J. Wang, S. Dodds, and W. Bailey, "Co-ordinated control of multiple robotic manipulators handling a common object; theory and experiments," IEE Proceedings-Control Theory and Applications, vol. 144, no. 1, pp. 73-86, 1997. 